\title{
Reducing Implicit Bias Through Curricular Interventions
}

\author{
Christy K. Boscardin, PhD \\ Department of Medicine, School of Medicine, University of California at San Francisco, San Francisco, CA, USA.
}

J Gen Intern Med 30(12):1726-8

DOI: $10.1007 / \mathrm{s} 11606-015-3496-y$

(c) Society of General Internal Medicine 2015

\begin{abstract}
A $\mathrm{s}$ an undergraduate at Berkeley, I was fortunate to live in International House, a multicultural residential complex of about 600 students from over 70 different countries that aims to foster intercultural respect through mutual understanding and tolerance. By witnessing and forming numerous positive intercultural friendships across political, religious, and social boundaries, International House completely reshaped how I viewed cultural differences as well as race relations in this country. Understanding the context for cultural differences as well as humanizing them ultimately promotes empathy and tolerance rather than validating stereotypes. The study featured in this month's issue of JGIM by Ryn et al. ${ }^{1}$ reminded me of how positive as well as negative social interactions can significantly change and modify the stereotypes we may hold unconsciously.
\end{abstract}

By 2035 , more than $40 \%$ of the US population will be comprised of minorities, making health inequity one of the most pressing policy issues facing our country. ${ }^{2}$ In contrast to explicit bias, implicit biases are the biases that are activated at the unconscious level or unintentionally and without one's awareness. As described by Malcolm Gladwell in his bestseller, Blink: "All of us have implicit biases to some degree. This does not necessarily mean we will act in an inappropriate or discriminatory manner, only that our first "blink" sends us certain information. Acknowledging and understanding this implicit response and its value and role is critical to informed decision-making and is particularly critical to those whose decisions must embody fairness and justice."3 Although multiple factors impact health disparities, previous research suggests that implicit bias may play a role in the ongoing health disparities in this country by contributing to differential treatment of patients based on race, gender, ethnicity and other social stereotypes.

Ryn et al. provide an interesting insight into the potential sources of change in students' implicit bias toward African Americans during medical school. They found that both informal (e.g., having heard attending physicians or residents make negative comments about African American patients and having had unfavorable contact with African American

Published online August 27, 2015 physicians) and formal (e.g., having completed a BlackWhite Implicit Association Test ${ }^{4}$ as part of implicit bias training) curriculum can either significantly increase or decrease implicit bias during medical school. One notable finding from this study is the significance of the informal (hidden) curriculum on students' implicit bias. A favorable interaction with African American faculty can help decrease implicit bias, whereas an unfavorable interaction can increase implicit bias. Given the paucity of research in successful interventions for changing implicit bias, understanding potential sources for implicit bias activation can help guide effective curricular interventions. As suggested by Ryn et al., to decrease implicit bias in health care, we need to target interventions at both the individual student level by increasing self-awareness, but also at the institutional level by changing the cultural environment. Using evidence from multiple domains of research in this area, I propose the following four recommendations: 1) increase self-awareness, 2) create an inclusive learning environment, 3) create learning opportunities for positive interaction, and 4) develop empathetic skills to decrease implicit bias. Although many of the ideas presented here have been discussed by other researchers in this field, ${ }^{5-7}$ these focused recommendations for curricular improvements will help maximize implementation success.

1) Increase self-awareness

Several studies suggest that cognitive overload, time pressure, and stressful environments can often be catalysts for activation of implicit bias. Inclusion of self-reflection exercises, including participation in implicit bias tests as part of the curriculum at all levels of the continuum from medical school through continuing medical education, may improve self-awareness and self-monitoring. Previous studies have shown that rather than suppressing automatic negative biases, a conscious acknowledgment of one's own biases and active efforts to refute those biases can have a positive impact. Given the consistent reporting of the positive impact of an implicit bias test on increasing self-awareness, instituting systematic efforts to help assess and monitor implicit bias among faculty and residents as part of their professional development would be critical to decreasing opportunities for negative associations. For example, being aware that negative emotions and stress can trigger social or racial stereotyping can help monitor and reduce potential implicit bias. Specifically for faculty and residents working with students, practicing 
reflective practice and role modeling these techniques can help to minimize activation of implicit bias, especially in an often stressful environment. Role-modeling more than formal course work in professionalism and cultural competency training is often cited as a major influence on students' development of high professional standards. Explicit instructions in stress reduction and other coping strategies such as cognitive restructuring can potentially help mitigate the stress level of a busy clinical environment.

2) Create an inclusive learning environment

One of the most significant ways to decrease implicit bias is by replacing existing automatic stereotypes with construction of new positive mental associations. Based on previous studies, some of the most desirable traits and descriptors of academic supervisors from students' perspectives include: approachable, inclusive, nonthreatening, inspiring, open-minded, encouraging, and credible (knowledgeable). For students to construct new positive associations, faculty and residents need to deliberately create an effective and safe learning environment that promotes inclusiveness. Informal curriculum can play a positive as well as negative impact on the socialization process of medical training. Approaching clinical learning environment from a social cultural perspective, where knowledge is co-constructed by the faculty and learners, it assumes that new schemas including new social schemas and associations are generated through shared experiences and opportunities. Using Lave and Wenger's ${ }^{8}$ communities of practice framework, learning can be conceived of as constructed through social and relational practices, making the invitational quality of faculty and residents to integrate students into a clinic team critical for effective socialization.

3) Increase opportunities for positive interaction Another approach to decreasing implicit bias is through more positive interactions with minority groups and opportunities to develop cultural competencies. Burgess et al. ${ }^{7}$ suggested that increasing provider confidence and ability to interact with socially dissimilar patients can increase opportunity to create positive associations to replace automatic negative stereotypes. There is a growing body of research supporting the positive impact of cultural competency training including field experience with community health and perspective taking exercises on intermediate outcomes such as knowledge, attitudes, and skills of health professionals. A variety of faculty development efforts including incorporation of interactive theater techniques by one institution helped increase faculty capacity to facilitate potentially challenging discussions on diversity and social justice issues with students. ${ }^{9}$ Boscardin et al. ${ }^{10}$ also found positive change in student attitudes' towards working with underserved populations with increased experience in competency training and community field work experiences.

4) Empathy skills development

Recent psychological research by Schumann et al. found that empathy towards racially dissimilar groups is malleable and can be modified by changing the mindset about empathy from a fixed trait to a skill set that can be improved with effort. ${ }^{11}$ Adopting this approach towards empathy provides a strong justification for greater emphasis on creating learning opportunities and skills development of these competencies for both students and faculty. Further research and investments in curricular innovations that provide opportunities for students to develop their empathetic skills through experiences such as patient navigators and advocates will not only increase their competencies, but also provide legitimate student roles as active agents in improving the culture of patient care.

Being forced to confront unconscious or conscious bias in a safe environment can have a profound impact on both interpersonal as well as professional relationships. From my own experience living in the International House, by allowing students from countries with political, military conflicts and cultural differences to cohabitate, share meals, and confront their own biases and understand the context for their biases fostered mutual understanding and respect as well as a breaking down of stereotypes, rather than creating more conflict. Borrowing the words of a fellow former resident and author, Amir Aczel, "At International House, I began to understand that the hatreds on which we had grown up were left far behind us, and that here... we could see one another as individuals, as people, as warm and caring human beings.",12

Corresponding Author: Christy K. Boscardin, PhD; Department of Medicine, School of Medicine, University of California at San Francisco, Box0710, 533 Parnassus Avenue, Suite U-80, San Francisco, CA 94143, USA (e-mail: christy.boscardin@ucsf.edu).

\section{REFERENCES}

1. van Ryn M HR, Phelan, SM, et al. Medical school experiences associated with change in implicit racial bias among 3547 students: A Medical Student CHANGES Study Report. J Gen Intern Med. 2015. doi:10.1007/s11606-015-3447-7.

2. Brach C, Fraser I. Can cultural competency reduce racial and ethnic health disparities? A review and conceptual model. Med Care Res Rev. 2000;57(Suppl 1):181-217.

3. Gladwell M. Blink :the power of thinking without thinking New York ittle, Brown and Co.; 2005.

4. Greenwald AG, McGhee DE, Schwartz JKL. Measuring individual differences in implicit cognition: The Implicit Association Test. J Pers Soc Psychol. 1998;74:1464-80.

5. Chapman EN, Kaatz A, Carnes M. Physicians and implicit bias: how doctors may unwittingly perpetuate health care disparities. J Gen Intern Med. 2013;28(11):1504-10.

6. Blair IV, Steiner JF, Havranek EP. Unconscious (implicit) bias and health disparities: where do we go from here? Perm J. 2011;15(2):71-8. 
7. Burgess D, van Ryn M, Dovidio J, Saha S. Reducing racial bias among health care providers: lessons from social-cognitive psychology. J Gen Intern Med. 2007;22(6):882-7.

8. Lave J, Wenger E. Situated Learning: Legitimate Peripheral Participation. Cambridge: Cambridge University Press; 1991.

9. Kumagai AK, White CB, Ross PT, Purkiss JA, O'neal CM, Steiger JA "Use of interactive theater for faculty development in multicultural medical education.” Medical teacher. 2007;29(4):335-40.
10. Boscardin CK, Grbic D, Grumbach K, O'Sullivan P. Educational and individual factors associated with positive change in and reaffirmation of medical students' intention to practice in underserved areas. Acad Med. 2014;89(11):1490-6.

11. Schumann K, Zaki J, Dweck CS. Addressing the empathy deficit: beliefs about the malleability of empathy predict effortful responses when empathy is challenging. J Pers Soc Psychol. 2014;107(3):475-93.

12. Aczel A. [cited July 20, 2015]; Available from: http://ihouse.berkeley.edu/ alumni/notable/ 